\title{
1 An open-source tool for the validation of finite element models using
}

\section{2 three-dimensional full-field measurements}

4 Alexander Abel ${ }^{\mathrm{a}}$, Stephanie L. Kahmann ${ }^{\mathrm{a}, \mathrm{b}}$, Stephen Mellon ${ }^{\mathrm{b}}$, Manfred Staat ${ }^{\mathrm{a}}$, $5 \quad$ Alexander Jung, ${ }^{\mathrm{a}, \text { ** }}$

6 Institute of Bioengineering, FH Aachen University of Applied Sciences, Heinrich-Mußmann

$7 \quad$ Straße 1, 52428 Jülich, Germany

$8 \quad{ }^{b}$ Nuffield Department of Orthopaedics, Rheumatology and Musculoskeletal Sciences, University

9 of Oxford, Windmill Road, Oxford, OX3 7LD, United Kingdom

$10 *$ *Corresponding author. Tel: +49 2416009 53861, E-mail address: a.jung@fh-aachen.de (A. Jung)

12 Abstract

13 Three-dimensional (3D) full-field measurements provide a comprehensive and accurate validation 14 of finite element (FE) models. For the validation, the result of the model and measurements are 15 compared based on two respective point-sets and this requires the point-sets to be registered in one coordinate system. Point-set registration is a non-convex optimization problem that has widely

17 been solved by the ordinary iterative closest point algorithm. However, this approach necessitates

18 a good initialization without which it easily returns a local optimum, i.e. an erroneous registration.

19 The globally optimal iterative closest point (Go-ICP) algorithm has overcome this drawback and

20 forms the basis for the presented open-source tool that can be used for the validation of FE models

21 using 3D full-field measurements. The capability of the tool is demonstrated using an application 
22 example from the field of biomechanics. Methodological problems that arise in real-world data

23 and the respective implemented solution approaches are discussed.

25 Keywords: 3D point-set registration; global optimization; digital image correlation (DIC);

26 biomechanics; synthetic bone

\section{Introduction}

31 Computational models, in particular finite element (FE) models, are increasingly being used to

32 understand and predict the behavior of complex biological systems, for which experimental

33 measurements are to some extent impractical or even impossible. However, any model is only a

34 simplified representation of the real world. For the model results to be (clinically) relevant, the

35 limitations of the model must be figured out and according to the National Academies of Sciences,

36 Engineering, and Medicine of the United States [1] and Viceconti et al. [2] three issues should be

37 addressed in particular: verification (confirming that the implementation of the model has been

38 done correctly and that the model equations are solved accurately), validation (determining how

39 well a model represents the real world phenomena it is intended to predict), uncertainty

40 quantification (determining how variations in the model affect its outcome). Validation is based

41 on the statistical comparisons of the model's outcome and experimental measurements and if

42 necessary, the model is modified in an iterative manner, e.g. by FE model updating [3], until the

43 difference between the prediction of the model and measurements satisfies the requirements. 
45 FE models are widely used in the field of biomechanics (e.g. [4]) and their validation is usually

46 based on displacements and/or strains measured on the surface of a given object under load. For

47 hard tissue, e.g. bone, strain gauges have been used for a long time [5]. Strain gauges are, however,

48 only capable of measuring strains at single spots in regions with very low strain gradients and they

49 reinforce materials with low Young's moduli [6] meaning strain readings can be error prone.

50 Contactless three-dimensional (3D) full-field measurement methods such as digital image

51 correlation (DIC) overcome these limitations [5][7] and allow for a more comprehensive and

52 accurate validation [8]. A speckle pattern is applied to the surface of the object and during loading

53 two cameras (or more) record images of the deforming object. The images are then divided into

54 groups of pixels (pixel-subsets) and the pixel-subsets in a reference image are correlated to the

55 same pixel-subsets in the following image in order to find the displacements for each subset center.

56 Spatial differentiation of the displacements can then be used to derive strains [9-10].

57

58 Displacements and strains are computed and measured pointwise. The FE point-set includes the 59 coordinates of the nodes of the mesh (FE points) and the DIC point-set includes the coordinates of 60 the pixel-subsets (DIC points). It is essential that the statistical comparison is done based on the 61 unloaded state of the object and that the point-sets are accurately registered in one coordinate 62 system. Point-set registration is a non-convex optimization problem, in which the spatial 63 transformation that minimizes the registration error has to be found. So far, the iterative closest 64 point (ICP) algorithm [11] has widely been used to solve the problem (e.g. [12-14]). The ICP 65 algorithm, however, requires a good initialization without which it easily converges on a local 66 minimum associated with an erroneous registration. This drawback has been overcome by the 67 globally optimal iterative closest point (Go-ICP) algorithm developed by Yang et al. [15]. 
69 The Go-ICP algorithm forms the basis of the presented open-source tool that is developed for the

70 validation of FE models using 3D full-field measurements. To the best of our knowledge, no other

71 such tool has been made available yet. The validation of a biomechanical model using DIC based

72 displacement measurements is used to describe its underlying methodology and to demonstrate its 73 capability in a proof-of-principle analysis.

74

\section{Structure of the tool}

\section{$76 \quad 2.1 \quad$ Framework}

77 The tool is written in Matlab (MathWorks, USA; it was tested for R2014a and later versions) and 78 runs on every computer platform. It requires the data to be sorted in columns and since there is no 79 standardized data format across FE and full-field measurement software, the integrated import 80 function in Matlab was selected for data import. This allows the individual selection of data 81 independent from formatting and column order. From that point on, the user is guided through the 82 entire validation process by a GUI dialogue.

83

$84 \quad 2.2 \quad$ Registration: Go-ICP

85 The point-sets are centered and normalized to the $[-1,1]^{3}$ space as this is a prerequisite for the 86 Go-ICP algorithm. The Go-ICP algorithm is a combination of the well-known ICP algorithm and 87 the Branch-and-Bound $(\mathrm{BnB})$ scheme and searches the spatial transformation that best aligns the 88 point-sets by minimizing the $L_{2}$-norm of the closest-point residual vectors (registration error $E$ ). 89 Full details can be found elsewhere [15] but a brief description is given here. 
91 Input for the algorithm are the two centered and normalized point-sets, a registration error $E$ set to

92 infinity, and an arbitrary initial transformation described by a rotation matrix and a translation

93 matrix. The BnB scheme starts by searching the space of $S E(3)$ for a transformation that gives an

94 upper bound value of $E$ which is lower than the current value of $E$. Having found a better

95 transformation, the ICP algorithm is called and initialized with that transformation. The ICP

96 algorithm then refines the transformation and the registration error $E$ can be further decreased.

97 This loop iterates until $E$ has converged to its global minimum $E^{*}$. The convergence threshold $\varepsilon=$

$98 a N$ depends linearly on the number $N$ of points; $a$ is the threshold factor. In the tool, the

99 transformation domain to be explored by Go-ICP is set to $[-\pi, \pi]^{3} \times[-1,1]^{3}$.

100

101 For equal point-sets, the Go-ICP has been proven to work reliably [15]. However, for several

102 reasons, point-sets obtained from real-world data are not equal and this is associated with an

103 inherent registration error and the emergence of additional local minima of $E$ that might be close

104 to the global minimum $E^{*}$. Thus, in order to find $E^{*}$, the convergence threshold needs to be small.

105 Starting out from a value of 0.001 , the threshold factor $a$ is iteratively decreased $(0.0005,0.0001$,

$1060.00005,0.00001, \ldots)$ until the registration error has converged to $E^{*} . E$ is defined to be converged

107 when the relative change between two consecutive iterations is between $0 \%$ and $5 \%$. To make the

108 registration process more robust to large closest-point distances (outlier), the tool also employs a

109 trimming procedure $[15,16]$ that removes a given percentage of points with largest closest-point

110 distances. Trimming is applied through a modification of the convergence threshold: $\varepsilon=a(1-$

$111 \rho$ ) $N$ and $\rho$ is the trimming factor ranging from 0 (no points are removed) to 1 (all points are

112 removed). The user can set $\rho$ depending on the desired amount of points in the registration and 113 ultimately in the statistical comparison. 


\subsection{Statistical comparison}

116 After registration, computed and measured displacements are statistically compared. This can be

117 done based on the original point-sets but the user is also given the opportunity to define a region

118 of interest (ROI). The spatial resolution of the point-set is determined by the number of points in

119 the given ROI. The point-set with lower spatial resolution is defined to be the primary point-set,

120 the other one is defined to be the secondary point-set. Statistical comparison is then done between

121 each displacement of the primary point-set and the averaged displacement of a surrounding point-

122 cloud of the secondary point-set (averaging method) or between closest-point pairs (closest-point

123 pair method). The point-cloud is bounded by a sphere around a given point of the primary point-

124 set (Fig.1) and the radius of that sphere can be set in the original length unit; its normalization is

125 done by the tool. No weighting is used for the averaging. In the case that no points lie within a

126 given sphere, the respective basis point is not considered for statistical comparison. The user can

127 decide whether to include only surface nodes or also nodes inside the given object depending on

128 the chosen FE-point set.

129

130 Statistical comparison includes linear regression analysis with information about the coefficient of 131 determination $\left(R^{2}\right)$, the slope, and the offset of the best fit line. Furthermore, the mean absolute 132 error $(M A E)$, the mean absolute percentage error $(M A P E)$, the root mean square error $(R M S E)$, 133 and the root mean square percentage error $(R M S P E)$ are computed. This is in line with previous 134 work on validations of biomechanical FE models [2][12][13][14]. The tool also provides a color135 map on the primary point-set that shows the absolute difference between computed and measured 136 displacements as in [8]. This is useful to identify regions with a good or bad agreement between 
137 the model's outcome and measurements. Closest-point pairs are used for the computation of 138 differences.

\section{Proof-of-principle analysis}

\section{$141 \quad 3.1 \quad$ Material and methods}

142 Exemplarily, a biomechanical FE model of a synthetic femur with a cemented hip implant was 143 validated at a compressive load of $5 \mathrm{kN}$ using DIC based displacement measurements. In 144 particular, total displacements were chosen.

The specimen was prepared by an experienced surgeon and potted in a $10^{\circ}$ angle in both the frontal 147 and the sagittal plane [17] using a custom-made guide. Plastic steel epoxy (Devcon, USA) was used to pot the femur in a plastic cup (filling height of the epoxy: $65 \mathrm{~mm}$, diameter of the cup: 100 $\mathrm{mm}$, femoral condyles were in contact with the plastic cup). The cup was held by a stainless steel 150 cylinder which was bolted upon a series of adjustable fixtures which were clamped onto the base 151 of the mechanical testing machine (Dartec H10, Zwick Roell Group, GBR). Load was applied at 152 the ball of the implant through a cylinder with a spherical hole whose diameter is $2 \mathrm{~mm}$ larger than 153 the ball diameter (28 mm). Mechanical testing was displacement-controlled (Control Cube, Zwick 154 Roell Group, GBR) with a displacement rate of $2 \mathrm{~mm} / \mathrm{s}$ and load and displacement were recorded 155 at $250 \mathrm{~Hz}$. The synthetic human femur (\#3403; Sawbones, USA) is made of short fiber filled epoxy 156 resin (cortical bone) and solid rigid polyurethane foam (cancellous bone), the hip implant Exeter 157 V40 (Stryker, GBR) is made of ultra-high strength stainless steel, the vacuum-mixed bone cement 158 Optipac (Biomet, GBR) is made of polymethyl methacrylate. 
DIC measurements were performed with the Q-400 system with two video cameras (resolution: 5 MP, frame rate: $10 \mathrm{fps}$ ) and the Istra 4D software (LIMESS Messtechnik und Software GmbH, 162 GER). For calibration, the calibration plate GL-06-WMB_9x9 from the same company was used. 163 Correlation parameters (subset size, spacing between the subset centers) were optimized by a 164 variation study with the criterion of obtaining minimum measurement error. The measurement 165 error was quantified by the total displacement averaged over all subsets for ten measurements 166 without load (where theoretical total displacements are zero). Minimum measurement error $167(0.0057 \mathrm{~mm})$ was obtained when the subset size was $19 \times 19$ pixels and spacing between the subset 168 centers was 4 pixels. Two filters (local regression and smoothing spline) were tested but their improvement on the measurement error (4\% and 6\%) was negligible small so that none of them 170 were applied. The optimized setting resulted in $N=11376$ subsets in the ROI (Fig.2a).

172 The FE model was developed with reverse engineered geometries of the synthetic femur and the 173 hip implant (SolidWorks, Dassault Systémes, FRA). The design of the cement mantle and the 174 positioning of the model parts were performed in line with X-ray images of the specimen. Linear 175 elastic transversely isotropic material properties (Tab.1) were assigned for the synthetic cortical 176 bone and linear elastic isotropic material properties (Tab.2) were assigned for the other model 177 parts. Computations were performed with Abaqus 6.12 (Simulia, Dassault Systèmes, FRA). 178 Modified second-order ten node tetrahedral elements (C3D10M) were used for meshing. A mesh 179 sensitivity study was performed to find the number of elements in the mesh at which the total 180 displacement converges. The FE-ROI was chosen to be similar to the DIC-ROI and the optimized 181 mesh consists of 6284 surface nodes within the ROI (Fig.2b). 
183 The spatial resolution of the FE point-set (6284 points/ROI) was lower than the DIC point-set 184 (11376 points /ROI) and thus, the FE point-set was the primary point-set. The trimming factor $\rho$ 185 was set to 0 and 0.1 . Statistical comparison was done based on the averaging method and the 186 point-cloud radius was set to $1.0 \mathrm{~mm}$.

$188 \quad 3.2 \quad$ Results

189 When performing the registration without the use of trimming, the registration error $E$ first 190 increased slightly and then decreased with decreasing threshold factor $a$ until it finally converged to $E^{*}: 9.47(a=0.001) ; 9.48(a=0.0005) ; 0.58(a=0.0001) ; 0.56(a=0.00005)$. The minimum 192 registration error $E^{*}$ was smaller $(0.42)$ when trimming with a factor of $\rho=0.1$ was used, but the 193 number of iterations until convergence remained unaltered. Trimming reduced the number of 194 available points for the statistical comparison to $5656 \mathrm{FE}$ points and $10238 \mathrm{DIC}$. The registered 195 point-sets that were trimmed with $\rho=0.1$ were used for statistical comparison. The FE model 196 predicted the measured total displacement with a coefficient of determination $R^{2}=0.85$, a slope 197 of 0.80 , and an offset of $-0.33 \mathrm{~mm}$. Errors were $M A E=0.93 \mathrm{~mm}, M A P E=30.60 \% R M S E=$ $1980.04 \mathrm{~mm}, \mathrm{RMSPE}=42.18 \%$, (Fig.3).

\section{Discussion}

202 We have introduced an open-source tool for the validation of FE models based on 3D full-field 203 measurements. For optimum point-set registration regardless of the initialization the Go-ICP 204 algorithm [15] was implemented and a GUI guides the user through the entire process. The 205 validation of a biomechanical synthetic bone-implant model based on DIC based displacement 
measurements was presented as an example to demonstrate the capability of the tool in a typical

207 scenario.

209 We used total displacements in the validation example because they were deemed more reliable than strains for the purposes of assessing the capabilities of the tool. The derivation of strains can

211 amplify errors contained in the displacements and in our case, large errors in strain measurements

212 were observed, which we attributed to noise [7][10]. However, the tool can be applied in the same 213 way to full-field strain measurements, which are generally more relevant for biomechanical studies 214 [21-22]. Moreover, the tool is suitable for application in other fields, e.g. thermodynamics, in 215 which FE models can be validated based on any kind of full-field measurements and furthermore, 216 the tool can also be used for comparing the outcome of two models with different meshes as part 217 of the verification and uncertainty quantification.

219 The Go-ICP algorithm has been proven to work reliably for equal point-sets [15] but point-sets 220 derived from real-world data differ from each other although they describe the same object. This 221 is e.g. because the sampling in full-field measurements cannot generate the exact same point-set 222 that is obtained by meshing in the FE model and because of deviations between the mesh and the 223 real object geometry. The setting of the convergence threshold becomes crucial because unequal 224 point-sets are associated with additional local minima that may lie close to the global minimum of 225 the registration error. Thus, we implemented a loop that decreases the convergence threshold 226 stepwise until it is small enough that the global minimum can be found. Outlier robustness of the 227 registration can be obtained by a trimming procedure [15-16]. However, as this procedure 228 decreases the number of points available for the statistical comparison, we suggest a careful setting 
229 of the trimming factor that gives a balanced trade-off between outlier robustness and amount of

230 available data for the statistical comparison. In the given example, the number and size of outliers

231 were small and thus, the registration error was not very sensitive to the trimming factor. After

232 registration, the values of the given primary point-set are statistically compared with the averaged

233 values of a surrounding point-cloud. This smoothing method was chosen to reduce the error that

234 might be introduced by large differences between computed and measured values when the 235 distance between a closest-point pair is high.

236

237 Perfect agreement between the model's outcome and full-field measurements would give a best fit 238 line with the $R^{2}$-value and the slope equal to 1 and the offset and the set of error measures equal 239 to 0 . However, in reality there are always errors. The main sources of errors in the FE model are 240 the geometry modelling and meshing, the constitutive model, and the boundary conditions. The 241 main sources of errors in full-field measurements depend on the particular application. This is, in 242 DIC measurements, the speckle pattern, the image acquisition and digitalization, and the 243 correlation parameter settings [5][7][10].

245 In the current study we have presented an open-source tool which can be used to validate FE 246 models based on full-field measurements. The tool was developed to provide a method of easily 247 linking computational and experimental research. Its capability has been tested in a typical 248 scenario and future research will utilize the tool in various biomechanical studies. 


\section{References}

255 [1] National Academies of Sciences, Engineering, and Medicine of the United States.

[2] Viceconti M, Olsen S, Nolte LP, Burton K. Extracting clinically relevant data from finite

[3] Avril S, Bretelle AS, Grédiac M, Hild F, Lenny P, Latourte F, Lemosse D, Pagano S,

Pagnacco E, Pierron F. Overview of identification methods of mechanical parameters based on full-field measurements. Exp. Mech 2008; 48: 381-402.

264 [4] Prendergast PJ. Finite element models in tissue mechanics and orthopaedic implant design.

[5] Grassi L, Isaksson H. Extracting accurate strain measurements in bone mechanics: a critical review of current methods. J Mech Behav Biomed Mater 2015; 50: 43-54. embedded in low modulus materials. Strain 2013; 49(4): 366-376.

[7] Palanca M, Tozzi G, Cristofolini L. The use of digital image correlation in the biomechanical area: a review. Int Biomech 2016; 3(1): 1-21.

272 [8] Gröning, F., Bright, J.A., Fagan, M.J., O’Higgins, P. Improving the validation of finite element models with quantitative full-field strain comparisons. J Biomech 2012, 45(8): 1498-1506. 
275 [9] Peters WH, Ranson, WF. Digital image techniques in experimental stress analysis. Opt Eng 1982; 21(3): 427-431.

277 [10] Pan B, Qian K, Xie H, Asundi A. Two-dimensional digital image correlation for in-plane 278

[11] Besl P, McKay N. A method for registration of 3-D shapes. IEEE Trans Pattern Anal Mach 280

[12] Grassi L, Väänänen S.P, Amin Yavari S, Weinans H, Jurvelin JS, Zadpoor AA, Isaksson optical measurements. J Mech Behav Biomed Mater 2013; 21: 86-94.

[13] Grassi L, Väänänen SP, Ristinmaa M, Jurvelin JS, Isaksson H. How accurately can subjectspecific finite element models predict strains and strength of human femora? Investigation using full-field measurements. J Biomech 2016; 49(5): 802-806.

[14] Gustafson HM, Cripton PA., Ferguson SJ, Helgason B. Comparison of specimen-specific vertebral body finite element models with experimental digital image correlation measurements. J Mech Behav Biomed Mater 2017; 65: 801-807.

[15] Yang J, Li H, Campbell D, Jia Y. Go-ICP: a globally optimal solution to 3D ICP point-set registration. IEEE Trans Pattern Anal Mach Intell 2016; 38(11): 2241-2254.

[16] Chetverikov D, Stepanov D, Krsek P. Robust Euclidean alignment of 3D point sets: the trimmed iterative closest point algorithm. Image Vis Comput (2015); 23(3): 299-309.

[17] Bergmann G, Graichen F, Rohlmann A, Bender A, Heinlein B, Duda GN, Heller MO, Morlock MM. Realistic loads for testing hip implants. Biomed Mater Eng 2010; 20(2): 6575. 
297 [18] Krause W, Mathis RJ, Grimes LW. Fatigue properties of acrylic bone cement: S.N, P-N, and P-S-N data. J Biomed Mater Res 1998; 22 (3 Suppl): 221-244.

299 [19] Orr JF, Dunne NJ, Quinn JC. Shrinkage stresses in bone cement. Biomaterials 2003; $300 \quad 24(17): 2933-2940$.

301 [20] Nuño N, Groppetti R, Senin N. Static coefficient of friction between stainless steel and 302 PMMA used in cemented hip and knee implants. Clin Biomech (Bristol, Avon) 2006; 303 21(9): 956-962.

304 [21] Nalla RK, Kinney JH, Ritchie RO. Mechanistic fracture criteria for the failure of human 305 cortical bone. Nat Mater 2003; 2(3): 164-168.

306 [22] Schulte FA, Ruffoni D, Lambers FM, Christen D, Webster DJ, Kuhn G, Müller R. Local 307 mechanical stimuli regulate bone formation and resorption in mice at the tissue level. PLoS One 2013; 8(4): e62172. 